\title{
Nowhere Patients
}

\author{
Elena Tomba
}

Affective Disorders Program, Department of Psychology, University of Bologna, Bologna, Italy

When Sir Austin Bradford Hill transferred the methodology that had accumulated with randomized trials in agricultural sciences to the first double-blind placebocontrolled study in medicine [1], the scenario was dominated by the challenges that acute diseases entailed. Tuberculosis was widespread, there were many drug reports in the literature claiming effectiveness of drug therapy, and the amount of streptomycin that was available was limited [2]. Randomized controlled trials (RCTS) in medicine thus referred to an average patient who fulfilled the criteria for admission and, unlike in agricultural sciences, ignored the individual history of the patient [3]. There were good reasons for the choices: most clinical encounters were for acute diseases and patients were unlikely to have experienced many treatments before (such as repeated exposure to antibiotics).

Today, most of the clinical activities are concentrated on chronic disease or non-disease-specific complaints [4]. Yet, the standard randomized controlled trial design is still based on the acute disease model and ideally evaluates therapeutic effects in untreated patients who have a recent acute onset of their disturbances. This is in sharp contrast with the fact that particularly in psychopharmacology, the patient is likely to have experienced other treatments before and these treatments may actually modify the course and responsiveness of the individual patient [5]. From a clinical viewpoint, it is quite different to treat a patient with a major depressive episode who displayed positive responses to previous therapeutic trials and a patient who failed to respond to various adequate trials [5]. These failed trials may have both neurobiological [6] and psychological [7] consequences. In neurobiological terms, phenomena such as the oppositional model of tolerance may become operational [6]. In psychological terms, patients may interpret unfulfilled expectations about therapy as an indication that their symptoms were worse than originally thought. The mechanisms may be particularly pronounced with overselling the efficacy of treatment (the violation of expectation) [7].

There is limited awareness of the limitations of the traditional randomized controlled trial and few suggestions for alternative models have been made. An adaptive treatment strategy has been recently developed for adjusting a treatment plan to the changing status of an individual patient, taking into account both the history of previous treatments and the response to those treatments [4]. Another design involves the sequential model, i.e. the sequential combination of treatments, such as psychotherapy after pharmacotherapy in depression [8-10]. Therapeutic targets are not predetermined, but depend on the response of patients to the first course of treatment. The sequential model design allows to randomize patients who are already in treatment and to assign them to treat-

\section{KARGER}

Fax +41613061234

E-Mail karger@karger.ch

www.karger.com
(C) 2012 S. Karger AG, Basel

0033-3190/12/0812-0069\$38.00/0

Accessible online at:

www.karger.com/pps
Elena Tomba, $\mathrm{PhD}$

Department of Psychology

University of Bologna, Viale Berti Pichat 5

IT-40127 Bologna (Italy)

Tel. +39 0512091 823,E-Mail elena.tomba@unibo.it 
ment alternatives according to stages of development and individual history and not simply to disease classification [10]. A further improvement over the traditional randomized controlled trial lies in refinement of criteria for eligibility to enter a trial, an approach that may take into account also a patient's history. For instance, in a recent RCT on psychotherapy of cyclothymic disorder [11], patients who had received antidepressive drugs in the past 2 years were excluded, since this might have affected the course of disease in an unpredictable way.

The study by Fava et al. [12] introduces important innovations to the traditional model of RCT in psychopharmacology. Two hundred and twenty-five subjects with major depressive disorder that was unresponsive to previous antidepressant treatment were randomized to adjunctive treatment with low-dose $(2 \mathrm{mg}$ /day) aripiprazole or placebo. Low-dose aripiprazole did not differ significantly from placebo in its therapeutic response. This was the first trial that used the sequential parallel comparison design (SPCD) that was introduced several years ago [13, 14]. The SPCD consists of two phases of treatment. In the first phase, more patients are randomized to placebo (75\%) than to active treatment (25\%). In the second phase, aripiprazole is increased from 2 to $5 \mathrm{mg}$ in those who received it from the beginning, whereas a part of those who were on placebo in the first phase is switched to $2 \mathrm{mg}$ aripiprazole. A further group continues on placebo. The analysis pools the data from both phases in order to maximize power, minimize the overall placebo response rate, and reduce the required sample size $[13,14]$. There are two important advantages of the design. One is that active treatment/placebo randomization in the second phase excludes not only the patients responding to the early effects of placebo, as would happen with the customary brief placebo washout periods, but also the patients who respond to unspecific ingredients (such as intensity of the patient clinician interaction), which may occur only at some later point in time [3]. The other advantage of the SPCD is the possibility of comparing not only patients between different groups (extensive design), but also within groups (intensive design), that is the same patients in two phases of treatment [14]. The design of this study [12] therefore maximized the likelihood of detecting significant differences between aripiprazole and placebo. Further, the assessment instruments that were selected, including both observer and self-related scale, were among the most sensitive that are currently available [15]. Yet, significant differences did not emerge [12]. This was attributed by the investigators essentially to the use of dosages that were lower than those that were em- ployed in other studies. However, an increase from 2 to $5 \mathrm{mg}$ did not seem to matter (a flat dose-response curve). There were also additional features that deserve attention. The diagnoses of major depressive episode was validated with specific criteria (SAFER) for improving the assessment accuracy of symptoms [16] that was likely to discriminate those symptoms that reflect the current state of illness and not long-standing traits or fluctuating mood. Further, the authors differentiated between inadequate response and relapse (loss of therapeutic response during initially successful antidepressant treatment). This latter neglected phenomenon appears to be a vexing problem with both antidepressant $[17,18]$ and secondgeneration antipsychotic $[19,20]$ drug treatment. Finally, the authors excluded patients with more than three episodes of unsatisfactory response to antidepressant therapy [12].

The following clinical vignettes may illustrate the clinical implications of these methodological choices. The 3 patients were referred to and treated in our Affective Disorders Program. They were all assessed with the Clinical Interview for Depression (CID) [21]. Response was defined as a reduction of at least $50 \%$ of the change version of the CID.

Mrs. A. was a 32-year-old married woman with a major depressive episode with melancholic features and paranoid delusions that was unresponsive to venlafaxine up to $300 \mathrm{mg} /$ day for 8 weeks. There was no previous history of mood disorders and use of antidepressant drugs. Addition of aripiprazole up to $20 \mathrm{mg} /$ day did not yield improvement in mood or delusional content. Switch to amitriptyline (up to $150 \mathrm{mg} /$ day) and perphenazine $(6 \mathrm{mg} /$ day) in combination yielded resolution of both depressive and paranoid symptoms. Perphenazine was tapered and discontinued. Mrs. A. was well, 1 year later, with $100 \mathrm{mg}$ of amitriptyline per day.

Mr. B. was a 27-year-old single man with a major depressive episode that did not respond to fluvoxamine up to $300 \mathrm{mg} / \mathrm{day}$ for 3 months. He was switched to mirtazapine up to $60 \mathrm{mg} /$ day for 6 weeks, but again this was not successful. Mr. B. had suffered from another episode 4 years earlier that had been successfully treated with paroxetine. In the current episode, he displayed considerable reactivity to social environment (changes in mood and circumstances), as rated by the CID [21]. Aripiprazole ( $2 \mathrm{mg}$ ) was added to mirtazapine and a satisfactory response was observed. Mr. B. then underwent psychotherapeutic treatment according to the sequential model $[9,10]$ and drug treatment was successfully tapered and discontinued. The patient was well at a 2-year follow-up. 
Mrs. C. was a 58-year-old married woman with a long history of recurrent major depressive episodes and obsessive-compulsive disorder. When she was first evaluated in the program, she was treated with fluvoxamine up to $200 \mathrm{mg} /$ day. Mrs. C. was also a long-time benzodiazepine user and at that time was taking alprazolam ( $4 \mathrm{mg} /$ day). She was switched to clobazam (40 mg/day). She responded to treatment with fluvoxamine and clobazam. Her response persisted for 1 year, when return of depressive and obsessive-compulsive symptoms ensued, despite cognitive behavioral treatment (CBT; 1 session every other week), which had been started in the meantime, and increase of fluvoxamine up to $300 \mathrm{mg} /$ day. Mrs. C. was then switched to clomipramine up to $150 \mathrm{mg} /$ day. After an excellent response, the clinical condition deteriorated in a few months. Addition of aripiprazole $10 \mathrm{mg} /$ day promptly yielded a response that, however, faded in a few weeks despite increase in dosage (up to $20 \mathrm{mg}$ ). Both clomipramine and aripiprazole were then rapidly decreased and substituted by amitriptyline (up to $150 \mathrm{mg} /$ day) and clonazepam ( $2 \mathrm{mg} /$ day). Mrs. C. responded to this combination and did not display loss of therapeutic effects at a 2-year follow-up, while continuing CBT.

The 3 cases share unresponsiveness to antidepressant drugs and show a variable response to adjunctive aripiprazole: lack of response for Mrs. A., good response for Mr. B., transient response for Mrs. C. What they do not share, however, is as important: no previous history of mood disorders and melancholic/psychotic features for
Mrs. A; one previous depressive episode and extreme reactivity to social environment potentially related to the long-term consequences of previous treatment with paroxetine [22] for Mr. B; obsessive-compulsive comorbidity and loss of therapeutic response for Mrs. C. Under ordinary conditions, these patients would all be eligible to be included in the same trial regardless of their treatment history. The heterogeneous features of these nowhere patients would then affect the outcome of the trial. Metaanalyses of these nowhere groups of patients may amplify the heterogeneous nature of the patient populations [23], particularly if random effects models were endorsed [24] and trials had different rates of participation [25]. Not surprisingly, the evidence that is provided by a single large trial appears to be less reliable than its statistical analysis suggests [26]. Borms and Donders [27] wondered whether treatments can better be evaluated by a series of small trials. Should treatment research in depression aim for large heterogeneous trials of nowhere patients or should we aim for small trials with very well defined treatment history? Should we limit observation to a month in the life of a patient or should we extend it to more prolonged periods of time? Should we limit assessment to the customary methods and symptoms, with their ensuing categorical definitions of remission, or expand assessment with broader strategies that have emerged in recent years [28-30]? These issues appear to be crucial for making true progress in depression treatment research.

\section{References}

1 Streptomycin in Tuberculosis Trials Committee: Streptomycin treatment of pulmonary tuberculosis. Br Med J 1948;ii:769-782.

$\checkmark 2$ Bradford Hill A: Memories of the British Streptomycin Trial in Tuberculosis. Control Clin Trials 1990;11:77-79.

-3 Fava GA: The intellectual crisis of psychiatric research. Psychother Psychosom 2006;75: 202-208.

$\checkmark 4$ Lavori PW, Dawson R: Adaptive treatment strategies in chronic disease. Annu Rev Med 2008;59:443-453.

5 Fava GA, Rafanelli R, Tomba E: The clinical process in psychiatry: a clinimetric approach. J Clin Psychiatry 2011, Epub ahead of print.

6 Tomba E, Offidani E, Fava GA: Selective serotonin reuptake inhibitors withdrawal syndromes: new insights into patophysiology and treatment; in Rees JP, Woodhouse OB (eds): Substance Withdrawal Syndrome. New York, Nova Science Publishers, 2009, pp 37-60.
7 Wolf S, Pinsky R: Effects of placebo administration and occurrence of toxic reactions. JAMA 1954;155:339-341.

$\checkmark 8$ Fava GA: Sequential treatment. Psychother Psychosom 1999;68:227-229.

-9 Tomba E, Fava GA: The sequential combination of pharmacotherapy and psychotherapy in mood disorders. J Contemp Psychother 2009;39:101-109

10 Fava GA, Tomba E: New modalities of assessment and treatment planning in depression. CNS Drugs 2010;24:453-465.

11 Fava GA, Rafanelli C, Tomba E, Guidi J, Grandi S: The sequential combination of cognitive behavioral treatment and well-being therapy in cyclothymic disorder. Psychother Psychosom 2011;80:136-143.
-12 Fava M, Mischoulon D, Iosifescu D, Witte J, Pencina M, Flynn M, Harper L, Levy M, Rickels K, Pollack M: A double-blind, placebo-controlled study of aripiprazole adjunctive to antidepressant therapy (ADT) among depressed outpatients with inadequate response to prior ADT (ADAPT-A study). Psychother Psychosom 2012;81:87-97.

13 Fava M, Evins AE, Dorer DJ, Schoenfeld DA: The problem of the placebo response in clinical trials for psychiatric disorders. Psychother Psychosom 2003;72:115-127.

14 Grandi S: The sequential parallel comparison model. Psychother Psychosom 2003;72: 113-114.

15 Tomba E, Fava GA: The emerging role of clinimetrics in psychological assessment; in Lange MA (ed): Leading-edge Psychological Tests and Testing Research. New York, Nova Science Publishing, 2007, pp 129-143. 
16 Targum SD, Pollack MH, Fava M: Redefining affective disorders: relevance for drug development. CNS Neurosci Ther 2008;14: 2-9.

-17 Fava GA, Offidani E: The mechanisms of tolerance in antidepressant action. Progr Neuropsychopharmacol Biol Psychiatry 2011;35: 1593-1602.

-18 Fabbri S, Fava GA, Rafanelli C, Tomba E: Family intervention approach to loss of clinical effect during long-term antidepressant treatment. Clin Psychiatry 2007;68:13481351.

19 Margolese HC, Chouinard G, Beauclair L, Bélanger MC: Therapeutic tolerance and rebound psychosis during quetiapine maintenance monotherapy in patients with schizophrenia and schizoaffective disorder. J Clin Psychopharmacol 2002;22:347-352.
20 Chouinard G, Chouinard VA: Atypical antipsychotics. Psychother Psychosom 2008;77: 69-77.

-21 Guidi J, Fava GA, Bech P, Paykel E: The clinical interview for depression. Psychother Psychosom 2011;80:10-27.

-22 Fava GA, Bernardi M, Tomba E, Rafanelli C: Effects of gradual discontinuation of selective serotonin reuptake inhibitors in panic disorder with agoraphobia. Int J Neuropsychopharmacol 2007;10:835-838.

23 Fava GA: Statistical alchemy for drug treatment of generalized anxiety disorder. Psychother Psychosom 2011;80:261-263.

24 Al khalaf MH, Thalib L, Doi SAR: Combining heterogeneous studies using the random-effects model is a mistake and leads to inconclusive meta-analyses. J Clin Epidemiol 2011;64:119-123.
25 Arfken CL, Balon R: Declining participation in research studies. Psychother Psychosom 2011;80:325-328.

26 Borm GF, Lemmers O, Fransen J, Donders R: The evidence provided by a single trial is less reliable than its statistical analysis suggests. J Clin Epidemiol 2009;62:711-715.

27 Borm GF, Donders R: A treatment should be evaluated by small trials. J Clin Epidemiol 2009;62:887-889.

28 Fava Ga, Sonino N, Wise TN (eds): The Psychosomatic Assessment: Strategies for Improving Clinical Practice. Basel, Karger, 2012.

29 Fava GA, Tomba E, Sonino N: Clinimetrics: the science of clinical measurements. Int J Clin Pract 2012;66:11-15.

30 Tomba E: Assessment of lifestyle in relation to health. Adv Psychosom Med 2012;32:7296. 